Supplementary information for:

\title{
Rapid Diagnosis of Prostate Cancer Disease Progression using Paper Spray Ionization Mass Spectrometry
}

Iqbal Mahmud ${ }^{\|}$, Frederico G. Pinto ${ }^{\dagger}$, Vanessa Y. Rubio», Bongyong Lee ${ }^{\perp+}$, Christian P.

Pavlovich $^{\Phi}$, Ranjan J. Perera ${ }^{\perp}$, and Timothy J. Garrett ${ }^{\| *}$

"Department of Pathology, Immunology, and Laboratory Medicine, University of Florida, Gainesville, Florida 32610, USA.

"Southeast Center for Integrated Metabolomics, Clinical and Translational Science Institute, University of Florida, Gainesville, Florida 32610, USA.

"University of Florida Health Cancer Center and UF Health, Gainesville, FL 32610, USA.

§Department of Chemistry, University of Florida, Gainesville, FL 32603, USA.

'Instituto de Ciencias Exatas e Tecnologicas, Universidade Federal de Vicosa, Vicosa 36570-900, Brazil

${ }^{\perp}$ Cancer and Blood Disorders Institute, Johns Hopkins All Children's Hospital, 600 6th Avenue South, St.

Petersburg, FL, 33701, USA.

${ }^{+}$Department of Oncology, Sydney Kimmel Cancer Center, Johns Hopkins University School of Medicine, 401 N. Broadway, Baltimore, MD, 21287, USA.

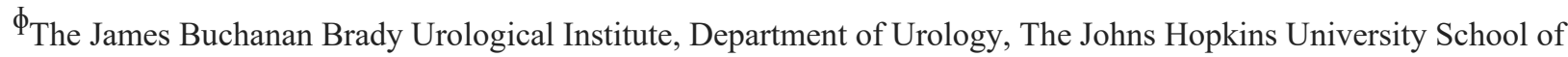
Medicine, 4940 Eastern Avenue, Baltimore, MD, 21224, USA.

Corresponding author: Timothy J. Garrett, Ph.D.

University of Florida

Gainesville, FL 32610

Email: tgarrett@ufl.edu

Phone: (352)273-5050

\section{Supplementary caption}

Figure S1. RSD calculation to show data reproducibility throughout the experiment.

Figure S2. Volcano plot-based differential regulated metabolic features in GS6 stage compared to healthy individuals.

Figure S3. Volcano plot-based differential regulated metabolic features in GS7 stage compared to healthy individuals.

Figure S4. Volcano plot-based differential regulated metabolic features in GS8 stage compared to healthy individuals.

Figure S5. Volcano plot-based differential regulated metabolic features in GS9 stage compared to healthy individuals.

Figure S6. Metabolic feature overlap between LC-MS and PSI-MS-based global metabolomics. 


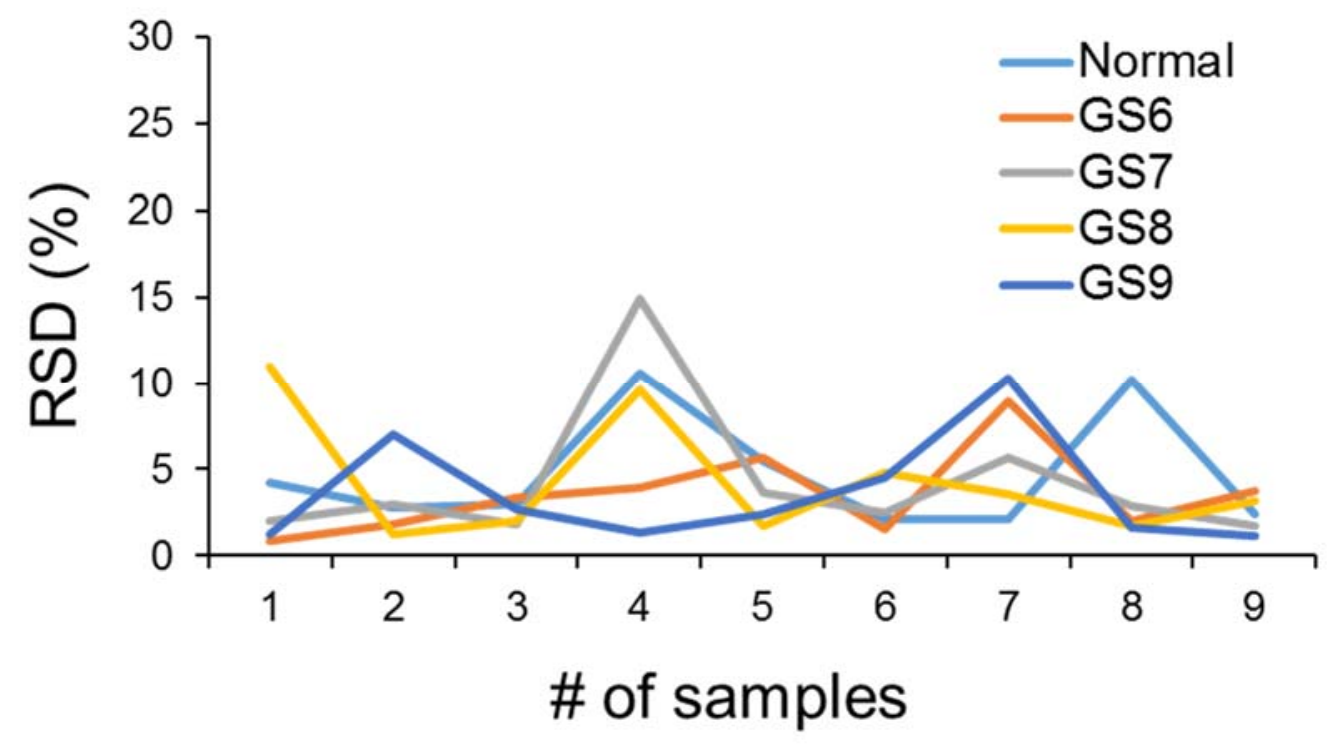

Figure S1. RSD calculation to show data reproducibility throughout the experiment. 


\section{GS6/N}

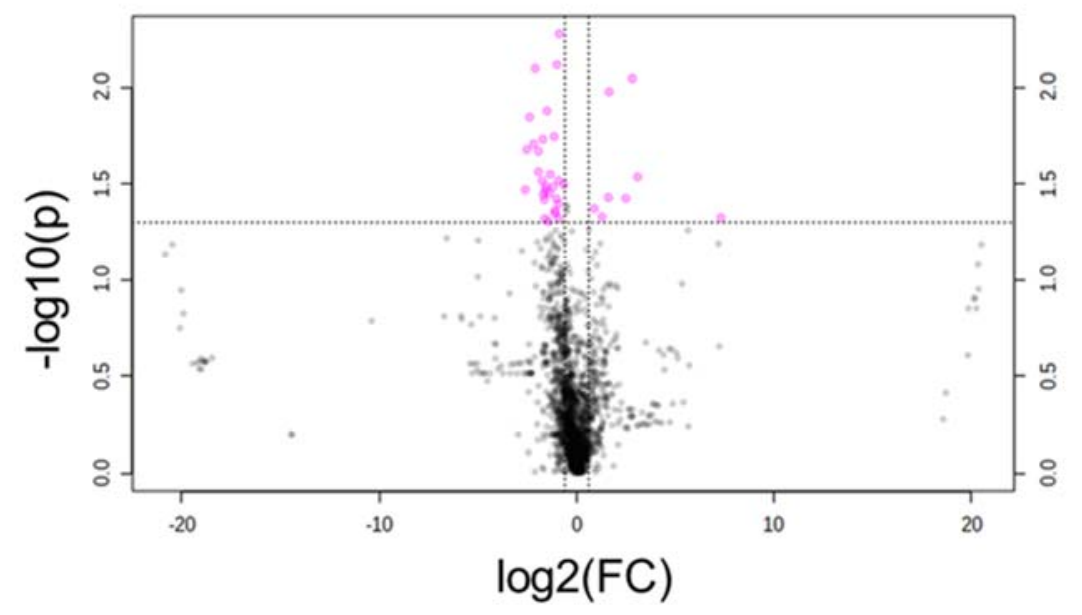

Figure S2. Volcano plot based differential regulated metabolic features in GS6 stage compared to healthy individuals.

\section{GS7/N}

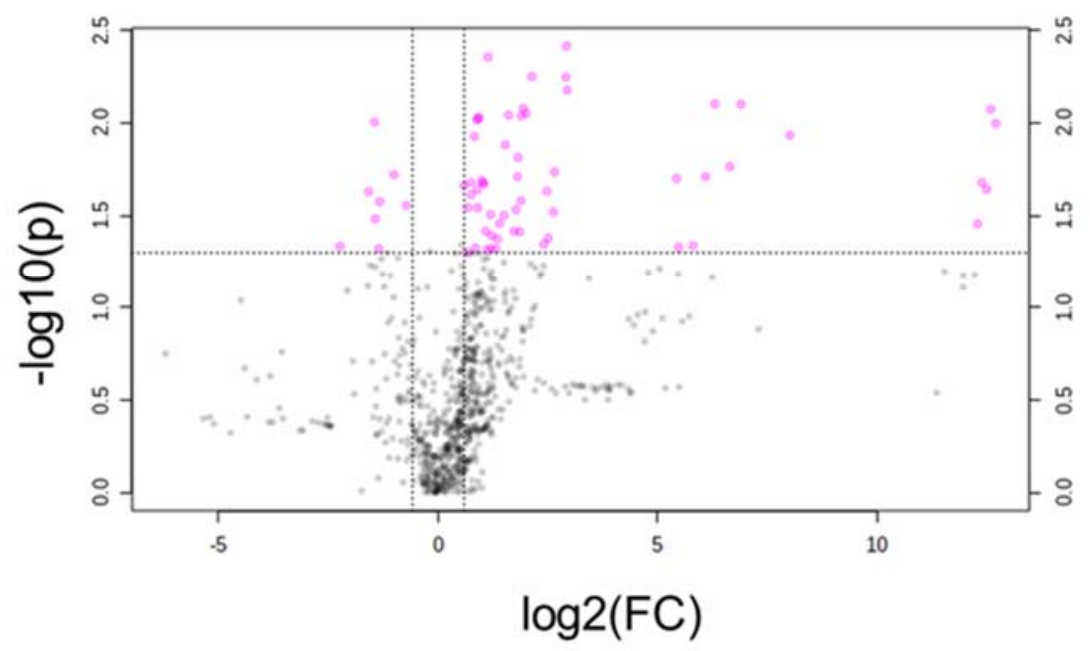

Figure S3. Volcano plot based differential regulated metabolic features in GS7 stage compared to healthy individuals. 


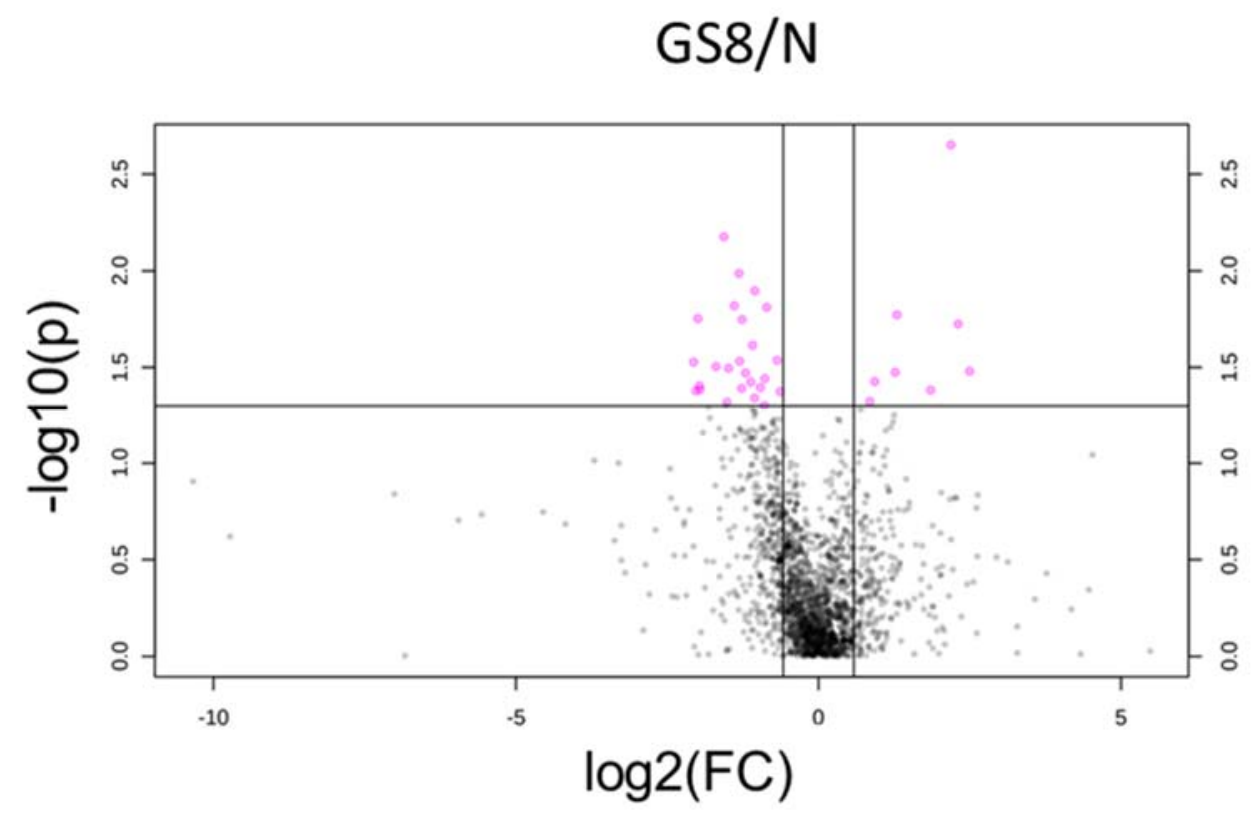

Figure S4. Volcano plot based differential regulated metabolic features in GS8 stage compared to healthy individuals.

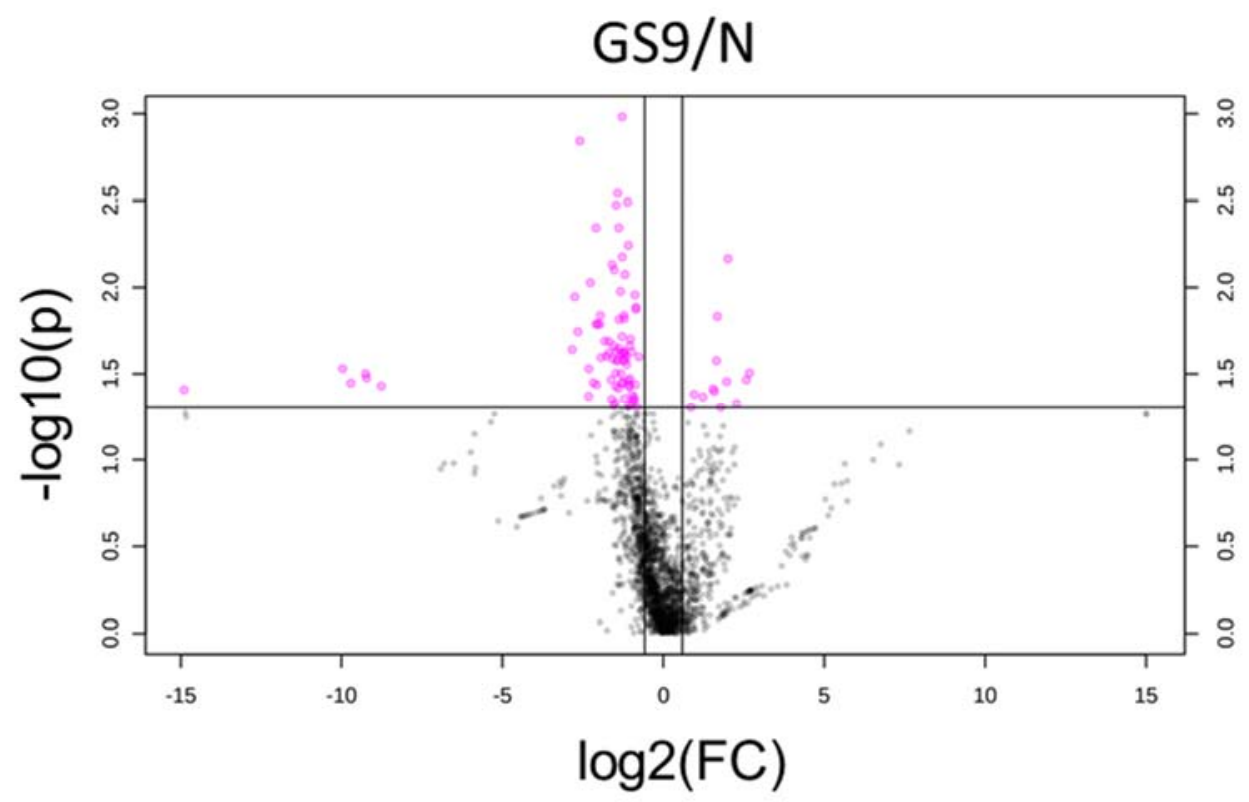

Figure S5. Volcano plot based differential regulated metabolic features in GS9 stage compared to healthy individuals. 


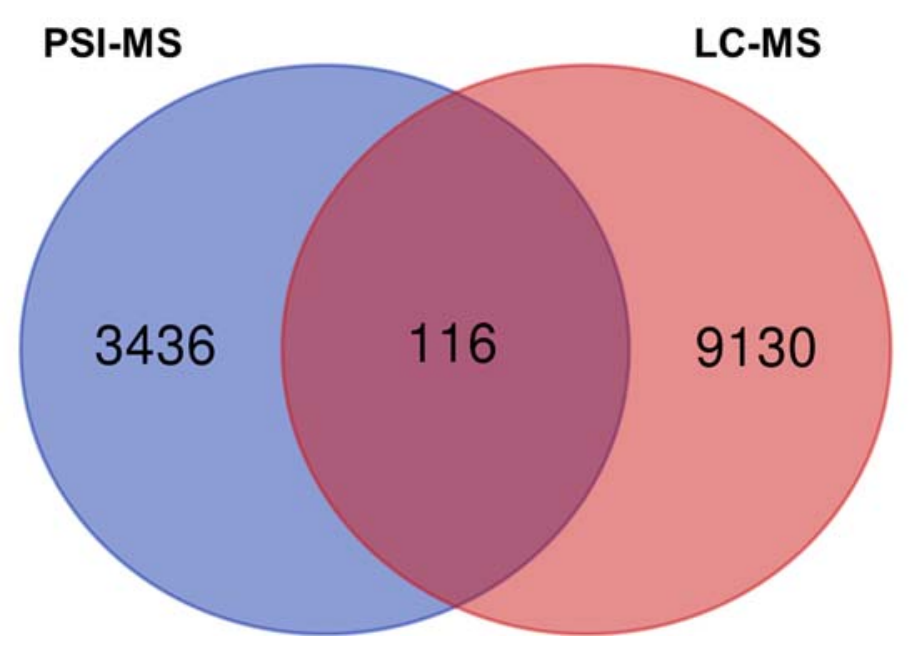

Figure S6. Metabolic feature overlap between LC-MS and PSI-MS-based global metabolomics. 